\title{
A Case of Recurrent Multiple Left Ventricular Thrombi without Thromboembolism in Noncompacted Myocardium
}

\author{
Teng Ye, Shusheng Liao* \\ The First Affiliated Hospital of Wenzhou Medical University, Wenzhou, China \\ Email: ^1007021910@qq.com
}

How to cite this paper: Ye, T. and Liao, S.S. (2019) A Case of Recurrent Multiple Left Ventricular Thrombi without Thromboembolism in Noncompacted Myocardium. Case Reports in Clinical Medicine, $\mathbf{8}$, 295-300.

https://doi.org/10.4236/crcm.2019.811036

Received: October 17, 2019

Accepted: November 22, 2019

Published: November 25, 2019

Copyright $\odot 2019$ by author(s) and Scientific Research Publishing Inc. This work is licensed under the Creative Commons Attribution International License (CC BY 4.0).

http://creativecommons.org/licenses/by/4.0/

\begin{abstract}
Background: Left ventricular noncompaction with multiple left ventricular thrombi can be revealed by echocardiography, and early diagnosis seems to be imperative to prevent significant embolic events. Case Report: A 57-year-old woman presented with symptoms of heart failure. Two-dimensional transthoracic echocardiogram demonstrated a dilated and diffusely hypokinetic left ventricle with severe impaired left ventricular systolic function. Moreover, a markedly thickened endocardium at the left ventricular apex and middle segment with numerous, excessively prominent trabeculations and deep intertrabecular recesses were present. During systole, the ratio of the noncompacted to compacted myocardial layers at the site of the maximal wall thickness was above two, a characteristic finding in left ventricular non-compaction. Multiple mobile, homogeneous, echodense thrombi were identified in the left ventricle, with the largest one in the apical noncompacted segment (dimensions, $32 \times 14 \mathrm{~mm}$ ). Cardiac magnetic resonance imaging confirmed the diagnosis of noncompacted myocardium with the presence of multiple thrombi. After anticoagulant therapy, her symptoms improved and thrombi dissolved. Unexpectedly, she re-admitted to the cardiovascular unit with progressive dyspnea. Transthoracic echocardiogram showed new large right atrial thrombi, with the largest one was $43 \times 38 \mathrm{~mm}$ compared to the echocardiogram done 11 months ago. The patient was anticoagulated with continuous heparin infusion for several days followed by oral Apixaban. After 4 weeks, the floating thrombi completely disappeared. After a 26-month follow-up, the patient's condition was stable without embolic complications. Conclusion: Echocardiography was the cornerstone of diagnostic methods for early detecting left ventricular thrombi to eventually prevent embolic events.
\end{abstract}




\section{Keywords}

Multiple Left Ventricular Thrombi, Noncompacted Myocardium

\section{Background}

Left ventricular noncompaction (LVNC) is an increasingly common cardiomyopathy characterized by the arrest of myocardial compaction during embryogenesis, leading to a non-compacted endocardial layer with marked trabeculations. Clinical presentation of Left ventricular noncompaction is highly variable. It can occur at any age, range from asymptomatic to end-stage heart failure, or be associated with lethal arrhythmias, sudden cardiac death, or thromboembolic events [1] [2] [3] [4] [5]. Although these intertrabecular recesses are prone to thrombus formation, early diagnosis as in our case seems to be imperative to prevent significant embolic events.

\section{Case Presentation}

A 57-year-old woman, presented to our hospital because of dyspnea, lower extremity edema, and fatigue symptoms. 24-hour Holter ECG monitoring showed left ventricular hypertrophy, a changed $\mathrm{T}$ wave with non-specific ST segment depression, no malignant arrhythmias were observed. Two-dimensional transthoracic echocardiogram (TTE) using a ultrasound scanner (PHILIPS iE Elite Ultrasound System) equipped with a $2.5-3.5 \mathrm{MHz}$ sector transducer demonstrated a dilated and diffusely hypokinetic left ventricle (end-diastolic/end-systolic dimension 59/47 $\mathrm{mm}$ ) with severe impaired left ventricular systolic function $(\mathrm{LVEF}=32 \%)$ and severe diastolic dysfunction $\left([\mathrm{E} / \mathrm{A}]\right.$ ratio $<2$; $\left.\mathrm{E} / \mathrm{E}^{\prime}=24\right)$. Moreover, a markedly thickened endocardium at the left ventricular apex and middle segment of the posterior and lateral walls with numerous, excessively prominent trabeculations and deep intertrabecular recesses was present. During systole, the ratio of the noncompacted to compacted myocardial layers at the site of the maximal wall thickness was above two, a characteristic finding in isolated left ventricular non-compaction. Doppler color flow mapping confirmed flow between the intertrabecular recesses. Multiple mobile, homogeneous, echodense thrombi were identified in the left ventricle, with the largest one in the apical noncompacted segment (dimensions, $32 \times 14 \mathrm{~mm}$ ) (Figure 1(A) \& Figure 1(B)). Color Doppler examination showed mild mitral regurgitation, moderate tricuspid regurgitation with mild pulmonary artery pressure (systolic pulmonary artery pressure $62 \mathrm{mmHg}$ ). The right atrial and right ventricular size and morphology were normal. There was no pericardial effusion. Cardiac magnetic resonance imaging (CMRI) confirmed the diagnosis of noncompaction of the myocardium with the presence of multiple thrombi (Figure 1(C)).

Then, she was immediately started on anticoagulant therapy for left ventricular thrombi, including intravenous Fraxiparine and subsequently oral Xarelto. One week later, she underwent a comprehensive echocardiographic approach, 

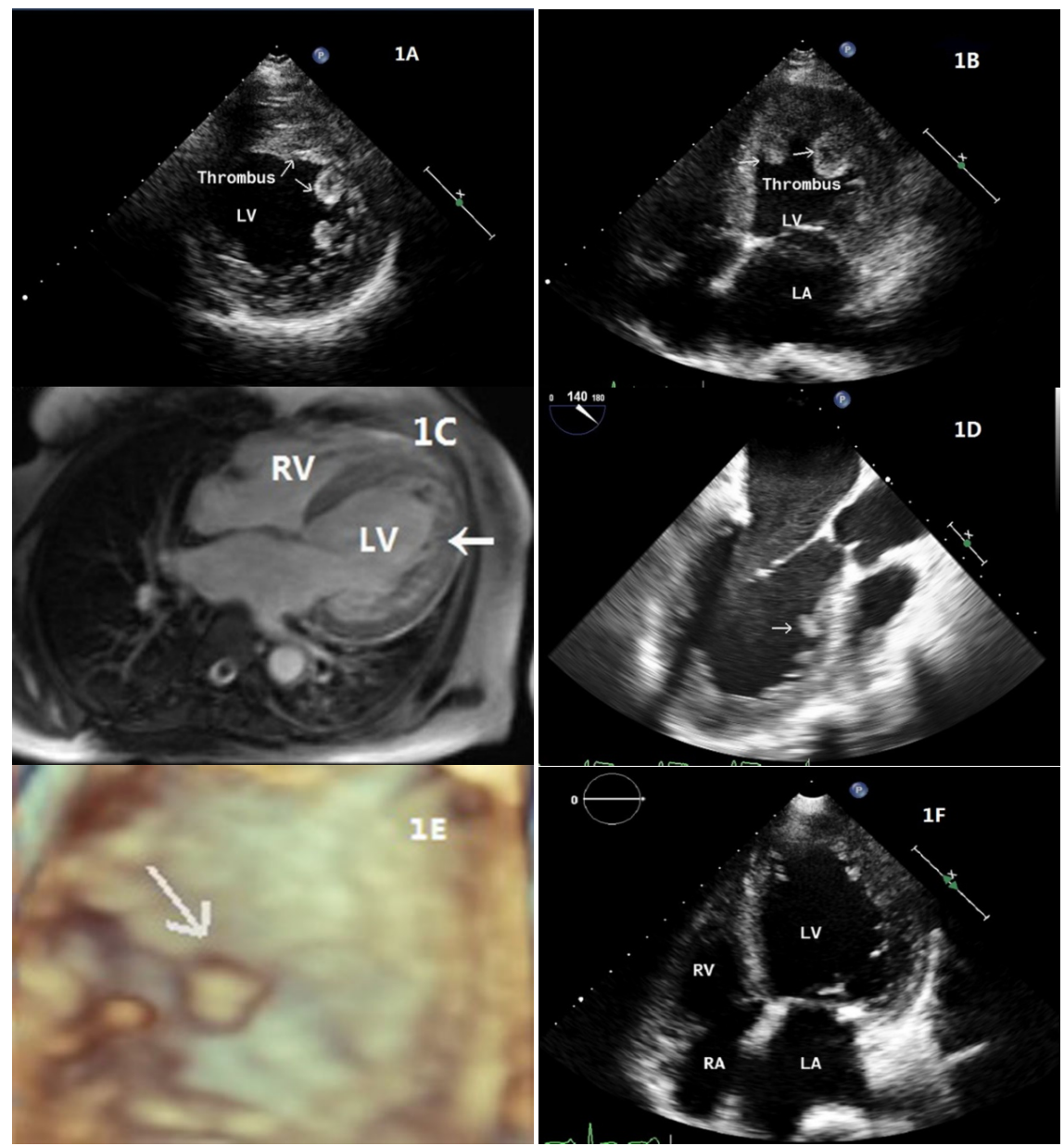

Figure 1. (A) Parasternal short-axis view showing multiple thrombi (arrows) in the mid segments of the anterior and lateral wall of the LV; (B) Apical 4-chamber view showing multiple thrombi (arrows) within the deep intertrabecular recesses at the apex and middle segment of the lateral wall; (C) CMRI showing left ventricular noncompaction in four-chamber; (D) Left ventricular long-axis view showing a small thrombotic mass (arrow) adjacent to the anterolateral papillary muscle; (E) 3D-TEE clearly delineating the thrombi (arrow); (F) Follow-up by 2D-TTE after 2 weeks anticoagulation therapy. Apical 4 -chamber views showing the noncompacted myocardium with absence of thrombi.

including transesophageal echocardiogram (TEE) (Figure 1(D)) and 3-dimensional imaging (Figure 1(E)), provided remarkably clear visualization of the hypertrabeculated myocardium, and a residual thrombus measuring $13 \times 9 \mathrm{~mm}$ was noted in the left ventricular apex.

The patient showed clinical improvement in her heart failure and was safely discharged on the thirteenth day of hospital admission. Left ventricular thrombi had resolved at this time (Figure $1(\mathrm{~F})$ ).

11 months later, she re-admitted to the cardiovascular unit with progressive dyspnea. Transthoracic echocardiogram showed multiple mobile thrombi were in the left ventricle, with the largest one in the apical noncompacted segment (dimensions, $43 \times 38 \mathrm{~mm}$ ). She was treated with continuous heparin infusion for several days followed by oral Apixaban. At the end of 4 weeks, follow-up trans- 
thoracic echocardiogram showed thrombi resolved. Low-dose digoxin was started on the day of discharged with a plan of up-titration the dose in subsequent weeks. The patient was currently in NYHA functional class III, with significant symptoms improvement, presented good therapeutic adherence and no need for hospitalization. She was referred for long-term anticoagulation with Apixaban in order to prevent any thromboembolic occurrences.

\section{Discussion}

In this case, recurrent multiple left ventricular thrombi in noncompacted myocardium occurred 2 times during one year despite anticoagulation therapy, but fortunately, without thromboembolism.

Left ventricular noncompaction is considered to be associated with a higher risk of in situ emboli than of other cardiomyopathies. Thromboembolism occurs in $20 \%-40 \%$ of cases and may involve the brain, lungs, and mesenteric organs [6]. The prominent ventricular trabecular meshwork and deep intertrabecular recesses causing stagnant blood flow, are importance structural basis for thrombi formation. The severe systolic dysfunction and enlarged left ventricle increases the risk of formation of multiple thrombus. Our patient presented with symptoms of heart failure secondary to left ventricular noncompaction and had multiple left ventricular thrombi. In this case, 2-dimensional transthoracic echocardiogram provided remarkably clear visualization of the hypertrabeculated myocardium, identifying mural thrombi wedged within the intertrabecular recesses, and led to a diagnosis of LVNC. Cardiac magnetic resonance imaging has been found to have very high specificity [7], suggesting effectiveness of cardiac magnetic resonance imaging as a confirmatory test after echocardiogram. With 3-dimensional transesophageal echocardiography, intracardiac masses can be sectioned using any desired angulation and viewed from multiple perspectives allowing comprehensive assessment of their size and mobility characteristics. Furthermore, thrombi were followed up conveniently by echocardiographic approach.

Unfortunately, routine use of anticoagulation to prevent thromboembolism in patients with LVNC remains controversial, and management of LVNC is mainly focused on control of symptoms and prevention of complications. Some authorities recommend anticoagulation in those with impaired systolic function (LVEF $<40 \%$ ) for the prevention of thromboembolism, although robust data are lacking to support this strategy [8]. In our experience, the incidence of multiple left ventricular thrombi in cases of noncompaction is low. Left ventricular systolic dysfunction in our patient might have increased the risk of in situ thrombi. Nonetheless, we think that chronic antiplatelet therapy with clopidogrel may not sufficiently prevent the formation of thrombi in this patient who have noncompaction and class III heart failure, which puts her at higher risk of systemic embolic event or sudden cardiac death in future. Given high risk of thrombi recurrence for such patient, clinicians should consider long-term oral anticoagulation 
for secondary prevention [9].

In conclusion, we describe a rare case of LVNC with multiple left ventricular thrombi but no thromboembolic events. This case illustrates the importance of early detection of left ventricular thrombi to eventually prevent embolic events and highlights the comprehensive role of echocardiogram in the evaluation of LVNC patients, leading in vivo detection of thrombi. Echocardiography plays a pivotal role as a first-choice technique of this abnormality because of its low cost and widespread availability and is the most frequently used first-line diagnostic tool for LVNC. Furthermore, our report emphasizes the need for anticoagulation when appropriate for the prevention of thromboembolic events associated with LVNC. However, to our knowledge, this case is only the second report [10] of direct visualization of thrombi without any evidence of embolic events in noncompacted myocardium. Combining different imaging techniques allow the clinician validation of findings and prevent reaching erroneous diagnosis which may have important consequences for the patient.

\section{Conclusion}

In conclusion, this case illustrates and reiterates the importance of echocardiogram in the detection of thrombus. Echocardiography was the cornerstone of diagnostic methods for early detecting left ventricular thrombi to eventually prevent embolic events.

\section{Conflicts of Interest}

The authors declare no conflicts of interest regarding the publication of this paper.

\section{References}

[1] Chin, T.K., Perloff, J.K., Williams, R.G., et al. (1990) Isolated Noncompaction of Left Ventricular Myocardium. A Study of Eight Cases. Circulation, 82, 507-513. https://doi.org/10.1161/01.CIR.82.2.507

[2] Oechslin, E. and Jenni, R. (2011) Left Ventricular Non-Compaction Revisited: A Distinct Phenotype with Genetic Heterogeneity. European Heart Journal, 32, 1446-1456. https://doi.org/10.1093/eurheartj/ehq508

[3] Pignatelli, R.H., McMahon, C.J., Dreyer, W.J., et al. (2003) Clinical Characterization of Left Ventricular Noncompaction in Children: A Relatively Common Form of Cardiomyopathy. Circulation, 108, 2672-2678. https://doi.org/10.1161/01.CIR.0000100664.10777.B8

[4] Towbin, J.A. (2010) Left Ventricular Noncompaction: A New form of Heart Failure. Heart Failure Clinics, 6, 453-469. https://doi.org/10.1016/j.hfc.2010.06.005

[5] Engberding, R., Yelbuz, T.M. and Breithardt, G. (2007) Isolated Noncompaction of the Left Ventricular Myocardium-A Review of the Literature Two Decades after the Initial Case Description. Clinical Research in Cardiology, 96, 481-488. https://doi.org/10.1007/s00392-007-0528-6

[6] Pitta, S., Thatai, D. and Afonso, L. (2007) Thromboembolic Complications of Left Ventricular Noncompaction: Case Report and Brief Review of the Literature. Jour- 
nal of Clinical Ultrasound, 35, 465-468. https://doi.org/10.1002/jcu.20349

[7] Petersen, S.E., Selvanayagam, J.B., Wiesmann, F., et al. (2005) Left Ventricular Non-Compaction: Insights from Cardiovascular Magnetic Resonance Imaging. Journal of the American College of Cardiology, 46, 101-105.

[8] Finsterer, J. (2010) Left Ventricular Non-Compaction and Its Cardiac and Neurologic Implications. Heart Failure Reviews, 15, 589-603. https://doi.org/10.1007/s10741-010-9175-5

[9] Thavendiranathan, P., Dahiya, A., Phelan, D., et al. (2013) Isolated Left Ventricular Non-Compaction Controversies in Diagnostic Criteria, Adverse Outcomes and Management. Heart, 99, 681-689. https://doi.org/10.1136/heartjnl-2012-302816

[10] Cevik, C., Shah, N., Wilson, J.M., et al. (2012) Multiple Left Ventricular Thrombi in a Patient with Left Ventricular Noncompaction. Texas Heart Institute Journal, 39, 550-553. 